\title{
An Interworking Call Control Solution for a Multidiscipline Switch
}

\author{
Pertti Raatikainen $^{1}$ and Sami Raatikainen ${ }^{2}$ \\ ${ }^{1}$ VTT Information Technology, Telecommunications, P.O. Box 1202, FIN-02044 VTT, \\ Finland \\ pertti.raatikainenevtt.fi \\ ${ }^{2}$ Helsinki University of Technology, Telecommunications Software and Multimedia \\ Laboratory, P.O. Box 5400, FIN-02015 HUT, Finland \\ sami.raatikainen@acta.fi
}

\begin{abstract}
The development of multimedia services is pushing networking technologies towards broadband solutions. A lot of research and development effort has been spent on finding a universal transport solution to carrying the different services to end-users. However, it looks that there will be no unifying transport solution to overcome the burden of networking diversity. This implies that the future services are delivered to users over heterogeneous networks. Therefore, interworking between the different networks and between the different signalling protocols becomes an essential part of the game. This paper introduces an interworking signalling solution for a switching equipment that integrates PDH time-slot and ATM cell switching. The switching platform is based on a multidiscipline switching fabric previously utilized in ATM switching applications.
\end{abstract}

\section{Introduction}

The rapid growth of Internet and advent of interactive multimedia communications is pushing the development of transport technologies towards heterogeneous networking. Services that traditionally have been carried over circuit or packet switched networks are seen to cross boundaries of the different network. This means that the future networks must be able to support services that have not originally been designed for them and, additionally, they must be able to communicate with other networks deploying different transport solutions. This places a major challenge for the equipment manufacturers and service providers. Manufacturers have to develop equipment having capabilities to interwork seamlessly with equipment supporting other networking technologies. Service providers have to satisfy a wide range of customer needs and try to guess the customers' future needs as well. So, a service provider needs an easy-to-extend environment.

End-users should be able to communicate with each other regardless of the underlying transport network. This means that the end-user needs universal access, i.e., access to other users as well as to all kinds of services. Therefore, network 
elements, such as switches, have to implement capabilities to communicate with different networks and support a diverse set of call and connection control schemes. The key issue, until all manufactures have the same technology if ever, is interworking.

Interworking between different networks and between different signalling protocols implies mappings and information conversions between the communicating parties. Both the hardware platform and the software framework have to implement different signalling protocol stacks with different signalling procedures and be able to interconnect them. In addition, different kinds of resource allocations and verifications, e.g., VPI/VCI and time-slots, have to be provided. Thus, a flexible framework is needed to enable all this in one software package.

In this paper, we introduce an interworking signalling solution for a switching equipment that integrates ATM (Asynchronous Transfer Mode) cell and PDH (Plesiochronous Digital Hierarchy) time-slot switching. The interworking is performed between narrowband and broadband signalling protocols, i.e., DSS1, ISUP, DSS2 and BISUP. The solution is based on an IN (Intelligent Networks) solution [10] that was considered an ideal basis for the work. The physical switching platform is based on a multidiscipline switch that integrates circuit, cell and packet switching into a single fabric [11]. The signalling architecture is based on the solution introduced in [12]. Chapter 2 introduces the IN concept and Chapter 3 presents the developed interworking software solution. Chapter 4 discusses the future work and Chapter 5 has the concluding remarks.

\section{IN Concept and Interworking}

Signalling protocols have evolved quite slowly along with terminals and network nodes. Development of ISDN networks introduced separate protocols for user and network signalling, because network nodes were assumed to be more intelligent than user terminals. The separation of signalling protocols based on different interfaces led to the need for mapping functions to transfer information between the involved protocols (e.g. DSS1 and ISUP) [13]. This can be seen as one form of interworking.

Interworking between the different networking technologies is a much more complex problem. In order to interconnect narrowband and broadband networks, more intelligent devices equipped with interworking capabilities are required. Even the networks implementing different protocol versions of the same signalling standard (e.g. ITU-T ISUP and ANSI ISUP) require interworking capabilities. Additionally, interworking between different interfaces within a certain type of network has to be supported.

Intelligent Networks (IN) [5] concept provides an open service platform where services can be located to selected network nodes and still are accessible from all corners of the network. The IN concept forms a multiservice environment enabling support for a large variety of connections with easily extensible IN services. The IN standards considered quite early interworking and suggested a general call model [5]. 
This environment can be utilized in realizing interworking between different sorts of networks.

The IN capabilities have successfully been deployed in today's digital telecommunications networks, such as ISDN (Integrated Services Digital Network), that utilize ITU-T's SS7 signaling (often implemented as a separate signalling network). Broadband technologies, such as ATM, can provide sophisticated control mechanisms and adequate transport capacity for a large set of services. Today, these technologies are mainly implemented in SDH (Synchronous Digital Hierarchy) based backbone networks, but with new and advanced access technologies, such as xDSL (Digital Subscriber Line), broadband services will become available to end-users who have a twisted pair connection to the trunk network. Figure 1 shows possible interconnections between different networks.

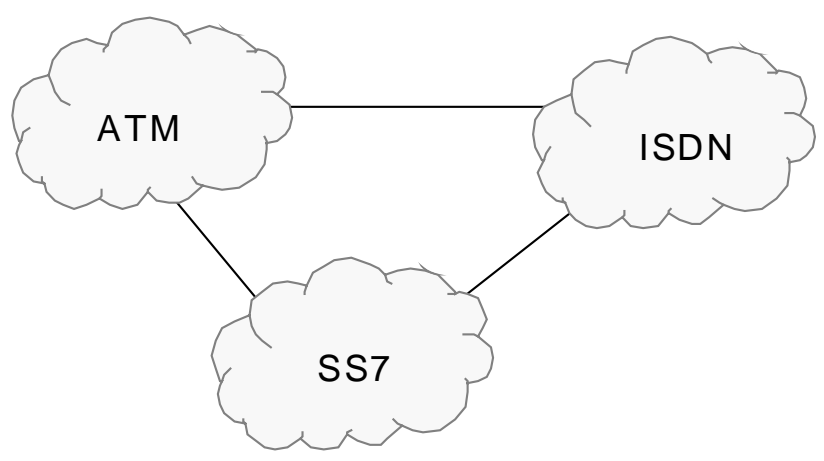

Fig. 1. Interconnections between different network technologies.

The IN concept defines a network service control architecture for open, distributed and service-independent communications. The architecture allows different services to be offered to customers independent of the underlying network technology. It also defines a service-oriented functional architecture that enables the provision of a set of generic service components of which new telecommunications services can be constructed. From the service point of view, the IN network can be considered as an overlay network, i.e., service control is separated from service switching functions.

The two basic components of the IN functional entities are Service Switching Point (SSP) and Service Control Point (SCP). SSP provides users with network access, performs switching functionality and allows users to access IN services. It detects IN service requests (e.g. a prefix of a number) and communicates with other physical IN entities. SSP contains Call Control Functions (CCF) and Service Switching Functions (SSF). CCF provides call/service processing and control. SSF provides a set of functions required for interaction between CCF and SCF (Service Control Functions). SCF is located in SCP that contains the service logic programs and data that are used in providing IN services. 
Examples of other functional entities are Intelligent Peripheral (IP) and Service Data Point (SDP). IP provides special resources with Specialized Resource Function (SRF). SDP contains Service Data Function (SDF) for real-time access to customer and network data. Figure 2 illustrates some of the IN entities.

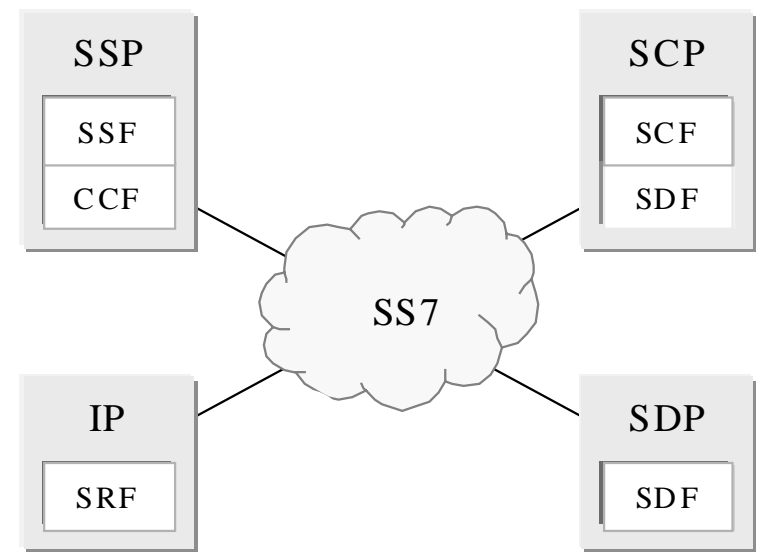

Fig. 2. Some physical entities of the IN architecture.

\section{Interworking Call Control Solution}

The ambitious goal of this work was to develop a modular software architecture, which ultimately enables an "any-to-any" interworking capability with any manufacturer's switch. The software can act as a SSP equipped with CCF and SSF functionality (see Fig. 2). It supports user signalling and bearer connection control at ISDN (Q.931) and at ATM (Q.2931, UNI3.1, UNI4.0) interfaces as well as network signalling at SS7 (ISUP) and ATM (B-ISUP) interfaces.

\subsection{Main Software Modules}

The interworking signalling implementation consists of software modules, compiled separately and linked together to form the entire software package. The major modules are the ICC (Interworking Call Control) module and the signalling stack modules (see Fig. 3). The ICC module includes the basic and generic CC (Call Control) and SCC (Switching Call Control) modules. The CC module implements a service switching point (SSP) which offers different call control functions (CCF), basically call models, for the originating $(\mathrm{CC}-\mathrm{O})$ and terminating (CC-T) sites of a call. The SCC module interconnects these call models and provides interworking functions and protocol specific bearer connection control functions. The FCF (Fabric Control Functions) module includes all the functions needed to control physical hardware connections. FCF utilizes API (Application Programming Interface) of the physical switch. 
The signalling stacks (DSS1, DSS2, ISUP and BISUP) function independently below the interworking call control layer. Each stack implements signalling and necessary lower layer protocols. The signalling stacks communicate with the call control application in the CC module via a common signalling interface, named SIGIF. Since the different signalling protocols use the same primitives, it has been possible to develop this common and well-defined "non-fat interface", primarily based on DSS1.

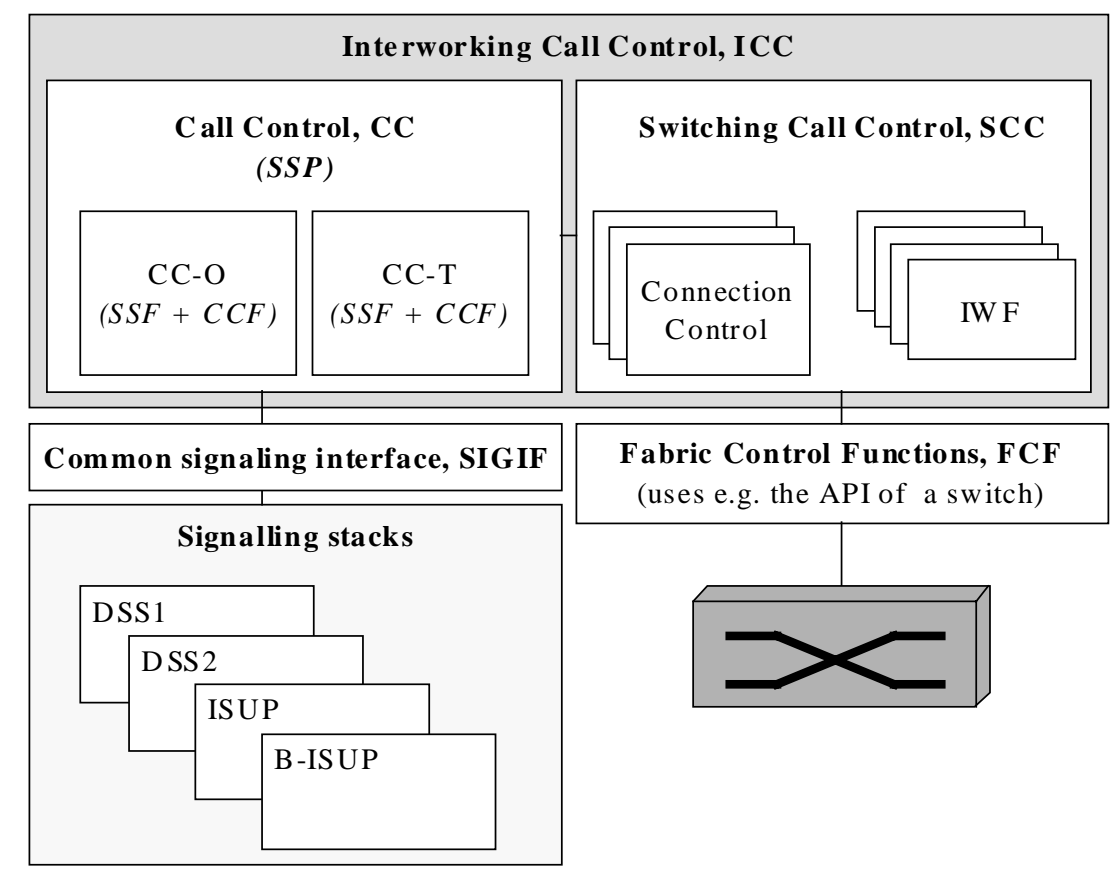

Fig. 3. Software modules.

\subsection{Interworking Call Control}

The interworking call control block, illustrated in Fig. 4, is composed of separate and independently interconnected modules for each subsystem (ATM, PDH and ISDN). Although these modules can be seen to function independently, they rely on other modules' support (e.g. SCC relies on the knowledge that a message sequence is checked by the signalling protocols).

The Call Control (CC) module, which is common to all signalling protocols and network types, can be considered as a central component. It is divided into two submodules $\mathrm{CC}-\mathrm{O}$ and $\mathrm{CC}-\mathrm{T}$. CC-O describes the call control model for all incoming calls and $\mathrm{CC}-\mathrm{T}$ the corresponding model for all outgoing calls. The implementation of these models is based on the definitions given in ITU-T's IN specifications [5]. SCC 
interconnects these two CC modules with the connection control and interworking functions. This implementation structure allows separation of call and connection control making the software more scaleable.

When a new call is requested, the required call control and interworking functions are activated. A "CC-O $<$ SCC $<>$ CC-T" connection is formed for each established call. Since $\mathrm{CC}-\mathrm{O}$ and $\mathrm{CC}-\mathrm{T}$ are common to all protocols, they retain the same structure for all calls. SCC, on the contrary, is created for each call individually and it describes all protocol and network dependent functions related to the call.

$\mathrm{CC}-\mathrm{O}$ and $\mathrm{CC}-\mathrm{T}$ process control information transparently which makes the $\mathrm{CC}$ module a generic one. This transparency is the primary reason for the need of the SIGIF interface. The CC module processes SIGIF primitives without deeper knowledge of their content; it knows only the primitive types and possibly some other information. The SCC module, on the other hand, knows all details of the SIGIF primitives and is capable of forming new ones with new parameters.

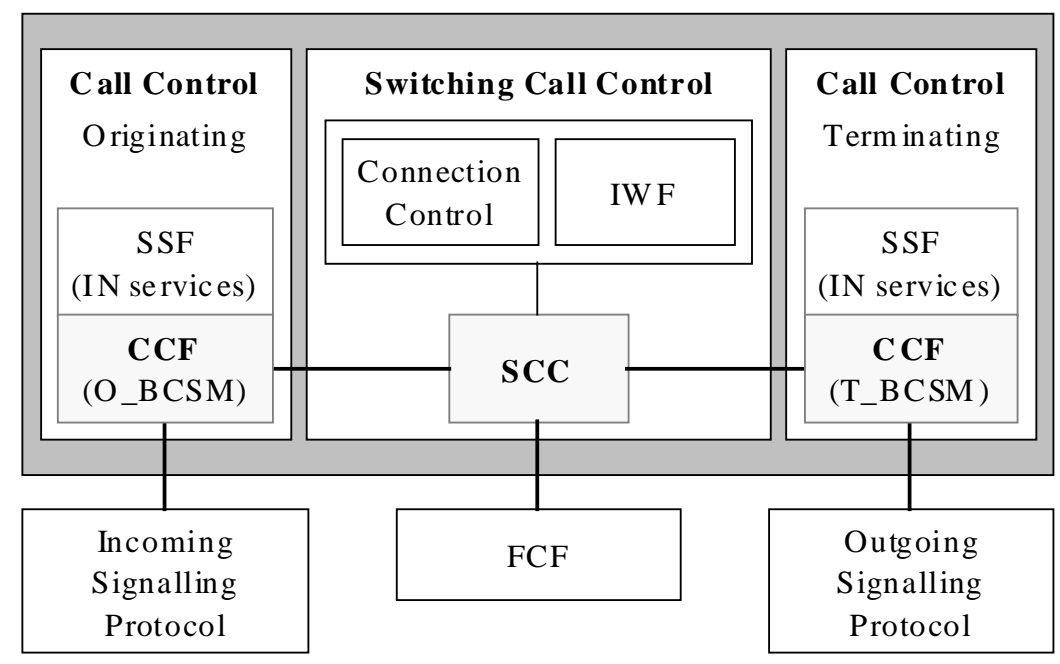

Fig. 4. Interworking call control.

The connection control functions in SCC know which methods to invoke in FCF to have the requested interconnection (e.g. to interconnect an ISDN channel with an ATM virtual channel). FCF "hides" the implementation of the physical switching fabric and hence a new fabric can be implemented just by adding a new FCF. By the use of this approach two FCF-solutions have been developed, one that uses GSMP (General Switch Management Protocol) [9] to manage a pure ATM switch and another that uses API of the deployed multidiscipline switch [11] to manage multiple switching schemes.

Since the interworking procedures depend on the originating site of the call and either end can function as the originator, different implementations are required for the 
two cases (see Fig. 5). All applied signalling protocols are based on ITU-T standards and thus the developed interworking solution is also based on ITU-T standards. Some modifications suggested by ETSI have been considered.

Interworking between the following signalling protocols (illustrated in Fig. 5) are supported:

1. Broadband interworking:

- $\quad$ DSS2 - BISUP $[1,6,8]$

2. Narrowband interworking:

- DSS1 - ISUP [4]

3. N-ISDN - B-ISDN interworking

- DSS1 - DSS2 [8]

- $\quad$ ISUP - BISUP $[2,7]$
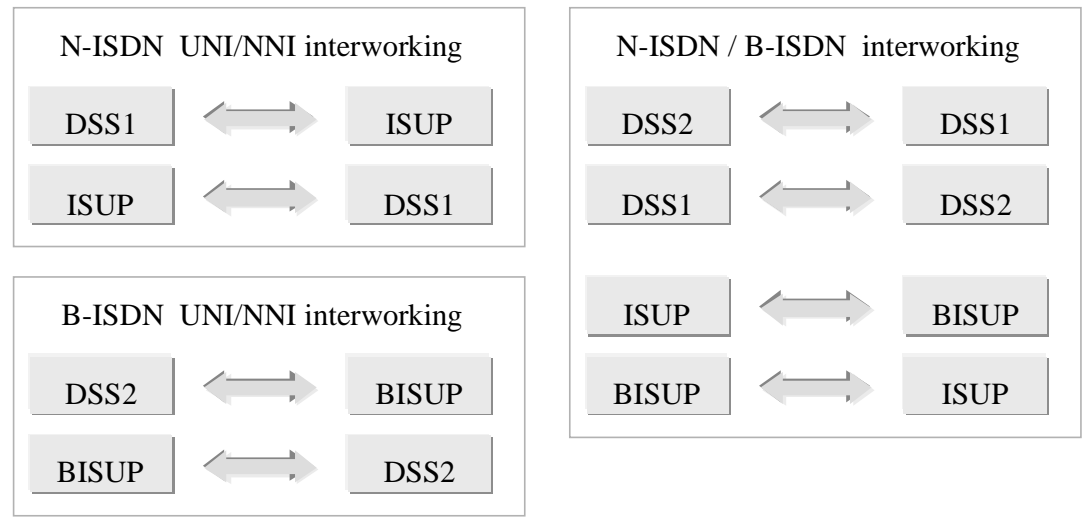

Fig. 5. Supported interworking procedures.

\subsection{Resource Allocation and Routing}

The starting point in the resource allocation was that a multidiscipline switch has to support different kinds of resources, e.g., ATM, conventional PDH and ISDN resources. All these with different traffic parameters have to be controlled which poses the problems of separating the resources and related procedures. In the case of interworking, different resources are tied to a connection and these should implement characteristics to support interoperability. Three resource entities (i.e. ATM, PDH and ISDN port type) were developed to describe port specific resources. They are used for controlling, e.g., ATM virtual channels, PDH time-slots and resources of the switching fabric. Each entity describes its own set of traffic characteristics and includes parameter lists and connection identifier tables, such as VPI/CVI tables. 
Routing refers here to routing of control messages and it may be static (i.e. routing tables configured by an operator) or dynamic (i.e. routing tables configured by a routing protocol). Routing has been implemented as a separate object, equipped with a unique interface and related functionality. This compact interface is relatively easy to include into an already existing routing solution. Thus, it is possible to implement a number of different routing solutions.

The SWITCH component is designed with a facade design pattern [3] and it provides a unified interface to all system resources. SWITCH controls all the system resources and routing operations as well as access to them (see Fig. 6). It is implemented using a singleton design pattern [3], which ensures that there exists only one instance of it and the software provides a global point of access to it.

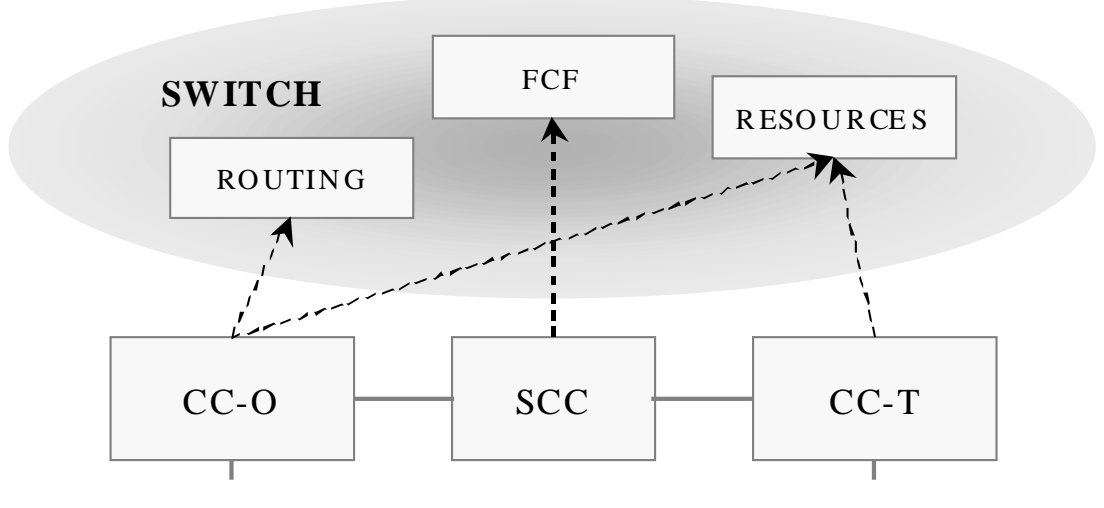

Fig. 6. Accessing services through the SWITCH component.

SWITCH initializes all software (including signalling stacks), controls numerous individual software components and directs incoming requests to relevant resource or routing entities. For example, a request coming from the $\mathrm{CC}$ module is directed to the relevant (i.e. ATM, PDH or ISDN) port entity, which reserves the needed resources (e.g. VPI/VCI). The resource control operations are hidden from the $\mathrm{CC}$ module, because only the port entities process the parameters of the connections. Thus call control can function independent of the physical switching fabric. When establishing or releasing connections, the SWITCH component provides the address of FCF and by using this address SCC can access functions needed to control the connections. The SWITCH component handless also routing requests and thus no other component can access routing resources.

\section{Future Work}

The deployed switching platform supports integration of ATM, PDH and IP communications and to have real convergence between these networks, interworking 
with IP networks have to be considered. Interworking with IP networks requires functionality to establish and control connections between IP and ATM or IP and PDH networks. This implies that software to implement "IP signalling" is needed. The developed modular architecture allows at least one way of adding IP signalling and connection control to the developed software. Fig. 7 illustrates integration of IP control to the developed software solution.

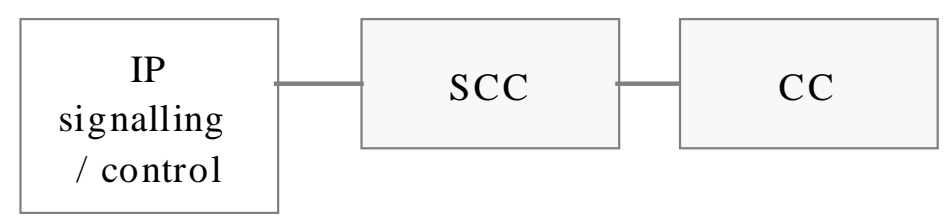

Fig. 7. Integrating IP control to the control software.

IP signalling and control module must include functions necessary for setting up and clearing connections also in the other networks involved with interworking, (i.e. ATM SVCs or PDH 64 kbps circuits). This module communicates with the SCC module, which will be able to convert and structure the information needed by the signalling protocol of the other communicating network. In the first phase, a connection would be needed from the IP switch control block to SCC. Then SCC could include functionality to make connections to the other network. Additionally, IP routing should also be implemented as a separate module.

\section{Conclusions}

Diversity in existing networking solutions and the development of multimedia services is leading to heterogeneity in networking. Since services are transported over different kinds of networks, it becomes essential to implement interworking facilities into the network. This article has introduced an interworking signaling solution for a switching equipment that integrates ATM cell and PDH time-slot switching.

The switching fabric is based on a switching solution, called SCOMS, that integrates ATM, conventional PDH and in the near future also IP switching into a single fabric. The key issue is to have reliable operation between the different systems and interchange signalling information. The developed system implements each subsystem separately, but the call control model is common to all integrated switching schemes.

The developed interworking solution utilises the general call models introduced in ITU-T's Intelligent Network (IN) concept. These models, defining separate call models for the originating and terminating site of a call, were adopted to our solution and every connection uses them in the same way. Modules implementing these call models function together as a Service Switching Point (SSP) and they are linked 
together with a Switching Call Control (SCC) module to enable interworking. A separate Fabric Control Function (FCF) was implemented to interpret control messages to the physical switch. The FCF module hides the physical switch implementation enabling a new fabric to be installed just by replacing the existing FCF by a new one.

The future work includes implementation of IP switching into the deployed multidiscipline switch platform. Separate software modules will be needed for routing and interworking solutions. Interworking requires implementation of "IP signalling" which must include capabilities to set up and clear connections also in the other networks that are involved with interworking.

\section{References}

1. ETSI ETS 300 495: Interworking between B-ISUP and DSS2.

2. ETSI ETS 300 496: Interworking between B-ISUP and ISUP.

3. Gamma, E., Helm, R., Johnson, R., Vlissides, J. Design Patterns: Elements of Reusable Object-Oriented Software. Addison-Wesley Publishing Company. Massachusetts (1995). ISBN 0-201-63361-2.

4. ITU-T Recommendation Q.699 (09/97): Interworking between ISDN access and non-ISDN access over ISDN User Part of Signalling System No. 7.

5. ITU-T Recommendation Q.1204 (03/93) - Intelligent network distributed functional plane architecture.

6. ITU-T Recommendation Q.2650 (02/95): Broadband-ISDN, interworking between Signalling System No. 7 - Broadband ISDN user part (B-ISUP) and Digital Subscriber Signalling System No. 2 (DSS 2).

7. ITU-T Recommendation Q.2660 (02/95): Broadband integrated services digital network (BISDN) - Interworking between Signalling System No. 7 - Broadband ISDN user part (BISUP) and Narrow-band ISDN user part (N-ISUP).

8. ITU-T Recommendation Q.2931 (02/95): Broadband Integrated Services Digital Network (B-ISDN) - Digital Subscriber Signalling System No. 2 (DSS 2) - User-Network Interface (UNI) - Layer 3 specification for basic call/connection control.

9. Newman P., Edwards W., Hinden R., Hoffman E., Liaw F. C., Lyon T., Minshall G.: Ipsilon's General Management Protocol - Version 1.1. IETF RFC 1987, Aug. 1996.

10. Puro V-M., Koponen P., Räsänen J., Nummisalo P., Martikainen O.: TOVE in Universal Mobile Telecommunications Systems. Proceedings of the 2nd Workshop on Personal Wireless Communications. IFIP TC6. Ed. By O. Spaniol, J. Slavik and O. Drobnik, Frankfurt am Main (Germany), Dec. 1996, pp. 103 - 111.

11. Raatikainen K. P. T.: Multidiscipline Switching for Delivering Multimedia Services. Proceedings of ICT'99. Cheju (Korea), June 1999, Vol. 2, pp. 86 -90.

12. Raatikainen K.P.T.: Interworking Support in a Multidiscipline Switch. Proceedings of Broadband Communications Conference 1999 (BC'99), Convergence of Network Technologies. Hong Kong, Nov. 1999, pp. 395 - 404.

13. Venieris I., Hussman H.: Intelligent Broadband Networks. John Wiley \& Sons (1998). ISBN 0-471-98094-3. 